\title{
IMPACT OF THE PERMEABLE WALL LINING ON THE PERISTALTIC FLOW OF WILLIAMSON FLUID IN AN INCLINED CHANNEL
}

\section{K. CHAKRADHAR, K. NANDAGOPAL \& T.V.A.P. SASTRY}

Department of Humanities \& Sciences, Kommuri Prathap reddy Institute of Technology, Hyderabad, Telangana. India

Department of Basic Sciences and Humanities, Sree Vidyanikethan Engineering College, A. Rangampet, Andhra Pradesh, India

Department of Humanities \& Sciences, Keshav Memorial Institute of Technology, Hyderabad, Telangana, India

ABSTRACT
The peristaltic flow of Williamson fluid in an inclined channel is studied. The walls of the canals are lined with a
porous material. The flow is generated due to the propagation of sine wave trains on the walls. To determine the model,
assumptions are made about the long wavelength and low Reynolds number. The solutions for the pressure gradient
and also the friction forces have been obtained for a diminutive Weissenberg number, by means of a disturbing
expansion around Weissenberg number. We also confer the graphical results of pressure increase, frictional force, and
time-averaged volumetric flow rate for an assortment of amplitude ratio values, permeability parameter, lining,
Weissenberg number, Reynolds number and angle of inclination.
Keywords: Williamson Fluid, Inclined Channel, Symmetric Channel, Thickness of Lining

Received: Jun 08, 2020; Accepted: Jun 28, 2020; Published: Sep 14, 2020; Paper Id.: IJMPERDJUN20201208

\section{INTRODUCTION}

Peristalsis, the apparatus for transporting fluid through porous channels, is an important method that allows many biological and mechanical processes, such as the transport of urine through the ureter, chyme into the oesophagus, the transmit of blood into the heart machine - lung and many others. Tang and Fung [1] and several many authors considered blood and other bio fluids behave like a Newtonian fluid for physiological peristalsis. Although the Newtonian blood methods give reasonable results for the ureter mechanism, it does not work for the small blood vessels and intestines. Recent studies indicate that the blood behavior is more likely non Newtonian (Majhi and Nair [2]). The blood in the small arteries and the fluids in the lymphatic vessels and intestines, urine in certain pathological conditions, etc., behave like non-Newtonian fluids. In addition, although the solution of nonNewtonian fluids is complicated by the appearance of the term non-linear, blood flow in the human body, explains the importance of the study of non-Newtonian fluids. Shapiro et al. [3], Srivastava and Srivastava [4], Vajravelu et al. [5], Kodandapani and srinivas [6], Srinivas and Gayatri [7] and others have studied the fluid mechanics of peristaltic pumping. The first quantitative approach to the peristaltic flow of non-Newtonian fluids was performed by Raju and Devanathan [8]. Mishra and Ghosh [9] considered the small blood vessels in the lungs to be porous channels and analyzed the distribution of pressure and velocity numerically. Several authors (Mishra and Rao [10], Abd. El. Hakeem et al. [11], Ebaid [12]) have considered the Newtonian fluid model for their study. Authors (Mekheimer [13]; Sobh [14]) advanced their research on peristaltic flow, considering uniform and non-uniform channels with different fluids and their corresponding parameters. Vajravelu et al. [15] studied the peristaltic 
transport of Williamson's fluid in an asymmetrical channel with permeable walls. Jayarami reddy et al. [16] studied the peristaltic flow of a Williamson fluid in an inclined plane channel under the influence of the magnetic field.

The flow through a porous medium was analyzed by the number of workers using Darcy's law. El Shahawey et al. [17] studied the peristaltic flow of generalized Newtonian fluid through a porous medium. Great interest in recent years El Shahawey et al.[18] examined peristaltic movement through a porous symmetrical channel. Ramesh and Devakar [19] analyzed peristaltic motion by considering the Williamson MHD fluid in an inclined asymmetric channel under heat transfer in a porous medium. Ellahi et al. [20] studied the application of peristaltic flow, in porous media and in a nonuniform channel. Manoranjan Mishra and Ramachandra Rao studied peristaltic transport in a two-dimensional channel filled with a porous medium in the peripheral region and a Newtonian fluid in the central region under long wavelength and low Reynolds number assumptions [21]. Sreenadh et al. [22] investigated the peristaltic flow of Herschel-Bulkley fluid in a flexible inclined channel lined with porous materials with a long wavelength and low Reynolds number count. Sankad [23] investigated the effect of permeable wall lining on peristaltic movement of Herschel Bulkley fluid flow, Reddappa et al. [24] investigated the peristaltic transport of Williamson fluid conduction in a porous channel. Bhatti et al. [25] studied biologically inspired heat transport on the flow rheology of Williamson's hydromagnetic nano fluids with convection.

In view of this peristaltic movement of Williamson fluid in an inclined channel was studied, according to assumptions of a low Reynolds number and long wavelength. The flow is studied in a wave frame that moves with the speed of the wave. The series of perturbations in the Weissenberg number was used to obtain explicit forms of the pressure gradient and the frictional force over a wavelength. The graphical result of the pressure increase, the friction force versus the mean volumetric flow over time were studied for different values of amplitude ratio, permeability parameter, lining value, Weissenberg number and pitch angle

\subsection{Nomenclatures}

- $\quad a$ Half width of the channel at the entrance.

- $\quad c$ wave speed

- $\quad D_{a}$ Darcy number

- $\lambda$ Wave length $p$ pressure

- $\bar{Q}$ Mean time of volumetric flow

- $\quad u$ axial velocity in the wave frame.

- $\varepsilon$ porous thickening of the wall

- $\tau_{y x}$ shear stress

- Re Reynolds number

- $\quad F r$ Froude number

- $\beta$ permeability parameter ratio of $D_{a}$ to $\sqrt{\alpha_{0}}$ 
- $Q$ Instantaneous volume flow rate

- $\quad F$ Friction force

- $\quad q$ Volume flux

- $\phi$ amplitude ratio

- $\quad \eta$ Ratio of Re to Fr

- $T$ time

- $\alpha_{0}$ slip parameter

- $\quad \alpha$ angle of Inclination

- $\Delta P$ pressure difference

- We Weissenberg number

\section{MATHEMATICAL FORMULATION}

Consider the peristaltic flow of Williamson fluid with walls lined by an inclined channel at an angle " $\alpha$ ". A train of peristaltic waves at speed " $c$ " is inserted into the walls of the channel. Channel width $2 a$. Here, the $\mathrm{X}$ axis is taken along the length of the channel and the $\mathrm{Y}$ axis is transverse. The physical model of that matter is shown in Figure 1.

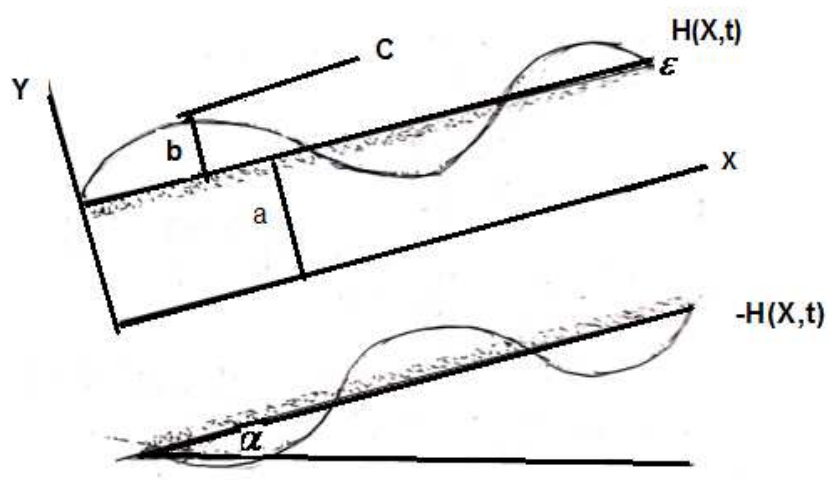

Figure 1: Physical Model.

The channel is governed by the equation

$$
Y=H(X, t)=a+b \sin \frac{2 \pi}{\lambda}(X-c t)
$$

Where $\lambda$ is the wavelength, $b$ as the amplitude of the wave and $a$ as half the width of the channel from the inlet at any axial distance, there is a constant flux in the frame of the wave $(x, y)$ moving with velocity " $c$ " away from the laboratory frame $(X, Y)$. However, in the coordinate system moving with the propagation speed " $c$ ", in the wave frame $(x, y)$ the shape of the boundary is stationary. 
The relationship between these two wave frames is given by

$$
\begin{aligned}
& x=X-c t ; y=Y ; u(x, y)=U(X-c t, Y)-c \\
& v(x, y)=V(X-c t, Y) ; p(x)=P(X, t)
\end{aligned}
$$

where $\mathrm{U}$ and $\mathrm{V}$ are components of the velocity in the laboratory frame and $\mathrm{u}, \mathrm{v}$ are components of the velocity in the wave frame. $p$ and $P$ are the pressures in waves and fixed reference systems, respectively. As demonstrated experimentally, Reynolds number of the flow is very small in many physiological situations. We assume inertial free flow with infinite wavelength. To formulate the basic equations and boundary conditions to be dimensionless, the following quantities are used:

$$
\begin{aligned}
& x^{\prime}=\frac{x}{\lambda} \quad y^{\prime}=\frac{y}{a} \quad h^{\prime}=\frac{h}{a} t^{\prime}=\frac{c t}{\lambda} \quad \varepsilon^{\prime}=\frac{\varepsilon}{a} ; \quad h=\frac{H}{a} \quad p^{\prime}=\frac{p a^{2}}{\eta_{0} \lambda c} \\
& q^{\prime}=\frac{q}{a c} u^{\prime}=\frac{u}{c} ; v^{1}=\frac{v}{c \delta} ; \quad \delta=\frac{a}{\lambda} ; \phi=\frac{b}{a} ; \quad D a=\frac{k}{a_{0}^{2}} ; \quad \operatorname{Re}=\frac{\rho a c}{\eta_{0}} \\
& \tau_{x x}^{\prime}=\frac{\lambda}{\eta_{0} c} \tau_{x x} \tau_{y x}^{\prime}=\frac{a}{\eta_{0} c} \tau_{y x} \tau_{y y}^{\prime}=\frac{\lambda}{\eta_{0} c} \tau_{y y} \quad F^{\prime}=\frac{F a}{\mu \lambda c} q^{\prime}=\frac{q}{a c} \\
& W e=\frac{\Gamma c}{a} ; F_{r}=\frac{c^{2}}{a g}
\end{aligned}
$$

Using the dimensionless quantities above and the resulting equations in terms of the velocity function can be written as

$$
\begin{aligned}
& \frac{\partial u}{\partial x}+\frac{\partial v}{\partial y}=0 \\
& \operatorname{Re} \delta\left(u \frac{\partial u}{\partial x}+v \frac{\partial u}{\partial y}\right)=-\frac{\partial p}{\partial x}-\delta^{2} \frac{\partial \tau_{x x}}{\partial x}-\frac{\partial \tau_{y x}}{\partial y}+\frac{\operatorname{Re}}{F r} \sin \alpha
\end{aligned}
$$

Where

$$
\tau_{x x}=-2(1+W e \gamma) \frac{\partial u}{\partial x} \text { and } \tau_{y x}=-(1+w e \gamma)\left(\frac{\partial u}{\partial y}+\delta^{2} \frac{\partial v}{\partial x}\right)
$$

Also $\delta, \operatorname{Re}, W e$ are wave, Reynolds, Weissenberg numbers respectively, after eliminating the primes and neglecting the terms of the order $\delta, \operatorname{Re}$, the governing equations are

$$
\frac{\partial}{\partial y}\left(\tau_{y x}\right)=-\frac{\partial p}{\partial x}+\eta \sin \alpha
$$


Where

$$
\tau_{y x}=\frac{\partial}{\partial y}\left[1+W e\left(\frac{\partial u}{\partial y}\right)\right] \frac{\partial u}{\partial y}
$$

The dimensionless boundary conditions are

$$
\begin{aligned}
& \frac{\partial u}{\partial y}=0 \quad \text { at } y=0 \\
& u=-1-\beta \frac{\partial u}{\partial y} \text { at } y=h-\varepsilon
\end{aligned}
$$

Where $\beta$ is permeability parameter, $\mathcal{E}$ is thickness of porous wall and $u$ is the velocity.

The volume of flow in each cross section is given by

$$
q=\int_{0}^{h-\varepsilon} u d y
$$

The volumetric flow rate $Q(X, t)$ between the center line and the channel wall is

$$
Q(X, t)=\int_{0}^{H} U(X, Y, t) d Y
$$

The average volumetric flow rate over time is

$$
\bar{Q}=\frac{1}{T} \int_{0}^{T} Q d t
$$

The pressure increase during a wave cycle is

$$
\Delta P=\int_{0}^{1}-\frac{\partial p}{\partial x} d x
$$

The dimensionless frictional force $F$ at a wavelength is

$$
F=\int_{0}^{1} h \frac{\partial p}{\partial x} d x
$$

\subsection{Solution}

Since equation (3) is a complex differential equation, it is difficult to get a solution. Therefore, we use the perturbation 
method. For this method we expand $u, q$ and $\frac{\partial p}{\partial x}$ as

$$
\begin{aligned}
& u=u_{0}+W e u_{1}+O\left(W e^{2}\right) \\
& \frac{\partial p}{\partial x}=\frac{\partial p_{0}}{\partial x}+W e \frac{\partial p_{1}}{\partial x}+O\left(W e^{2}\right) \\
& q=q_{0}+W e q_{1}+O\left(W e^{2}\right)
\end{aligned}
$$

Here Weissenberg number is so small ( We ), consider the first and second Systems only

\subsection{System of Order of $W e^{0}$}

$$
\frac{\partial}{\partial y}\left(\frac{\partial u_{0}}{\partial y}\right)=-\frac{\partial p_{0}}{\partial x}+\eta \sin \alpha
$$

The boundary conditions are

$$
\begin{aligned}
& \frac{\partial u_{0}}{\partial y}=0 \quad \text { at } y=0 \\
& u_{0}=-1-\beta \frac{\partial u_{0}}{\partial y} \text { at } y=h-\epsilon
\end{aligned}
$$

\subsection{System of Order of $W e^{1}$}

$$
\frac{\partial}{\partial y}\left(\frac{\partial u_{1}}{\partial y}\right)+\frac{\partial}{\partial y}\left(\frac{\partial u_{0}}{\partial y}\right)^{2}=-\frac{\partial p_{1}}{\partial x}
$$

The boundary conditions are

$$
\begin{aligned}
& \frac{\partial u_{1}}{\partial y}=0 \text { at } y=0 \\
& u_{1}=-\beta \frac{\partial u}{\partial y} \text { at } y=h-\epsilon
\end{aligned}
$$

Solving equations (2.15) using conditions of (2.16) we have

$$
\frac{\partial p_{0}}{\partial x}=-\frac{\left(q_{0}+h-\varepsilon\right)}{B_{2}}-\eta \sin \alpha
$$

Where 


$$
B_{1}=(h-\varepsilon)\left(\beta+\frac{h-\varepsilon}{2}\right) \text { and } B_{2}=\left(B_{1}-\frac{(h-\varepsilon)^{2}}{6}\right)(h-\varepsilon)
$$

Solving equation (19) using conditions (20) we have

$$
\frac{\partial p_{1}}{\partial x}=-\frac{\left[q_{1}-\left(\frac{\partial p_{0}}{\partial x}+\eta \sin \alpha\right) B_{4}\right]}{B_{2}}
$$

$$
\text { Where } B_{3}=(h-\varepsilon)^{2}\left(\beta+\frac{h-\varepsilon}{3}\right) \text { and } B_{4}=(h-\varepsilon)\left(B_{3}-\frac{(h-\varepsilon)^{3}}{12}\right)
$$

From (22) and (23) we have

$$
-\frac{\partial p}{\partial x}=\frac{(q+h-\varepsilon)\left(B_{2}+W e B_{4}\right)}{B_{2}^{2}}+\eta \sin \alpha
$$

The dimensionless pressure rise per wavelength defined as

$$
\Delta P=-\int_{0}^{1} \frac{\partial p}{\partial x} d x
$$

The dimensionless frictional force $F$ on the wall along a wavelength is

$$
F=\int_{0}^{1} h\left(\frac{\partial p}{\partial x}\right) d x
$$

Note: The results coincide with the results of paper in the reference (15) when angle of inclination and lining value tends to zero.

\section{RESULT ANALYSIS}

We presented the graphical result of the pressure increase $\Delta P$ and the frictional force $F$ with the mean volumetric flow over the respective time $\bar{Q}$ for different amplitude ratio values ' $\phi$ ', permeability parameter ' $\beta$ ', lining ' $\mathcal{E}$ ', Weissenberg number ' $W e$ ', Reynolds number ' $R e$ ', Froud number ' $F r$ ' and the angle of inclination ' $\alpha$ '. 


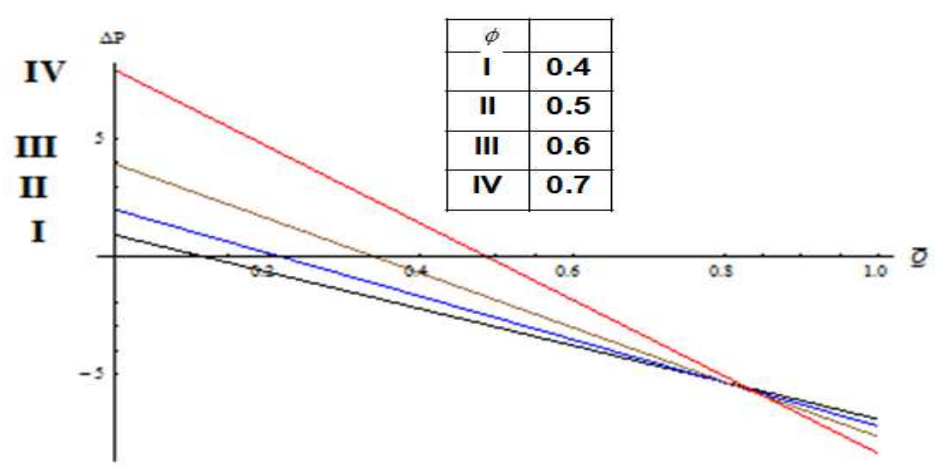

Figure 2: The Pressure Rise $\Delta P$ Variation with Mean Time of the Volumetric Flow Rate $\bar{Q}$ for Different Values of $\phi$ With

Fixed $\beta=0.3, \varepsilon=0.042, W e=3 \alpha=\frac{\pi}{6}$ And $\eta=1.25$.

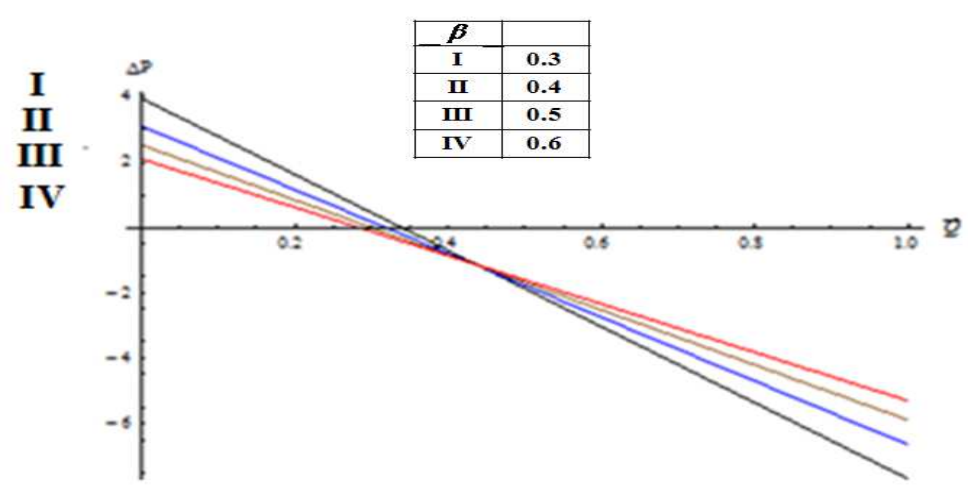

Figure 3: The Pressure Rise $\Delta P$ Variation with Mean Time of the Volumetric Flow Rate $\bar{Q}$ for Different Values of $\beta$ With

Fixed $\phi=0.6, \varepsilon=0.04, w e=3, \alpha=\frac{\pi}{6}$ And $\eta=1.25$.

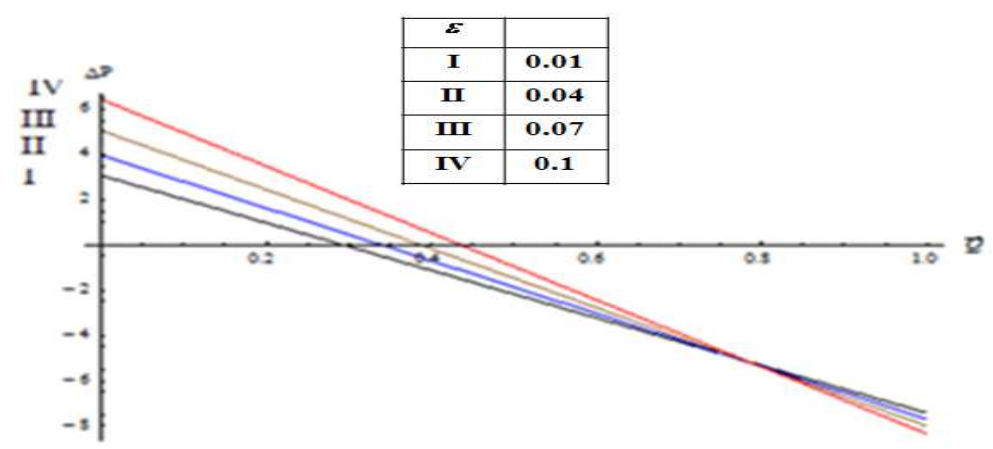

Figure 4: The Pressure Rise $\Delta P$ Variation with Mean Time of the Volumetric Flow Rate $\bar{Q}$ for Different Values of $\mathcal{E}$ With Fixed

$$
\phi=0.6, \beta=0.3, W e=3, \alpha=\frac{\pi}{6} \text { And } \eta=1.25 \text {. }
$$




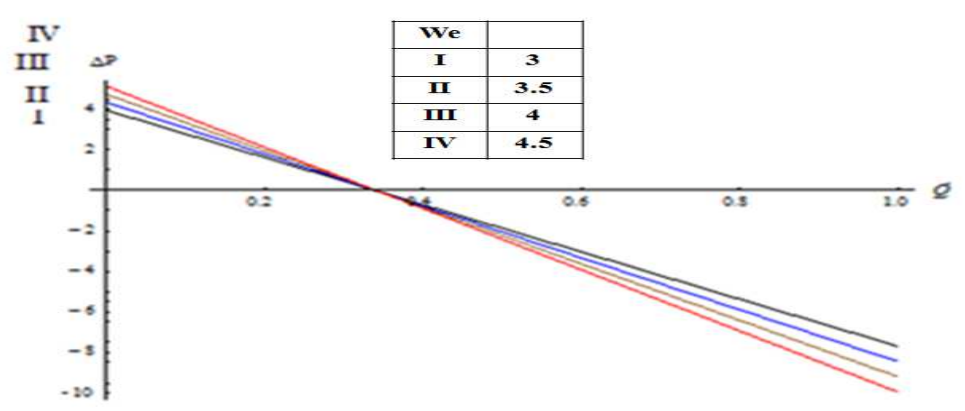

Figure 5: The Pressure Rise $\Delta P$ with Mean Time of the Volumetric Flow Rate $\bar{Q}$ for Different Values of $W e$ With Fixed $\phi=0.6, \beta=0.3, \varepsilon=0.04, \alpha=\frac{\pi}{6}$ And $\eta=1.25$.

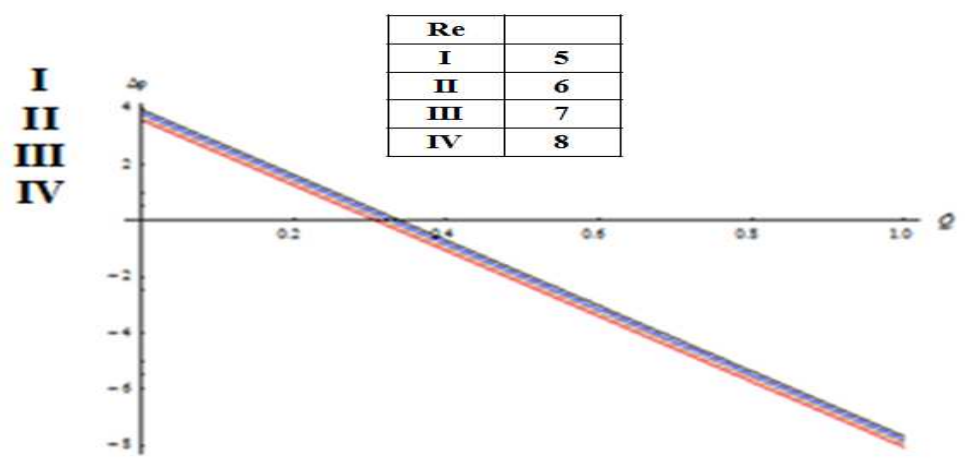

Figure 6: The Pressure Rise $\Delta P$ Variation With Mean Time of the Volumetric Flow Rate $\bar{Q}$ For Different Values Of Re With Fixed $\phi=0.6, W e=3 ; \beta=0.3, \varepsilon=0.04, \alpha=\frac{\pi}{6}$, And $F r=4$.

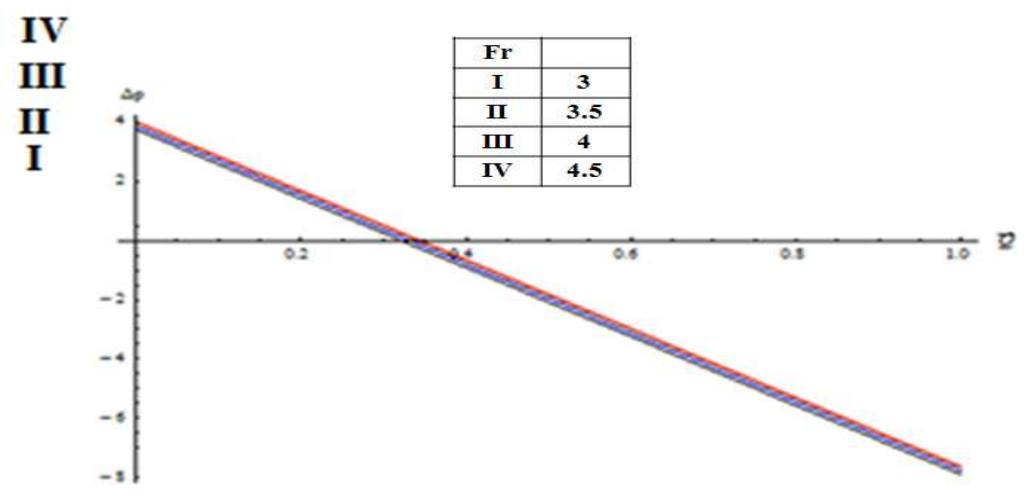

Figure 7: The Variation of Pressure Rise $\Delta P$ with Mean Time of the Volumetric Flow Rate $\bar{Q}$ for Different Values of $F r$ With Fixed

$$
\phi=0.6, w e=3 ; \beta=0.3, \varepsilon=0.04, \alpha=\frac{\pi}{6} \text {, and } \operatorname{Re}=5 \text {. }
$$




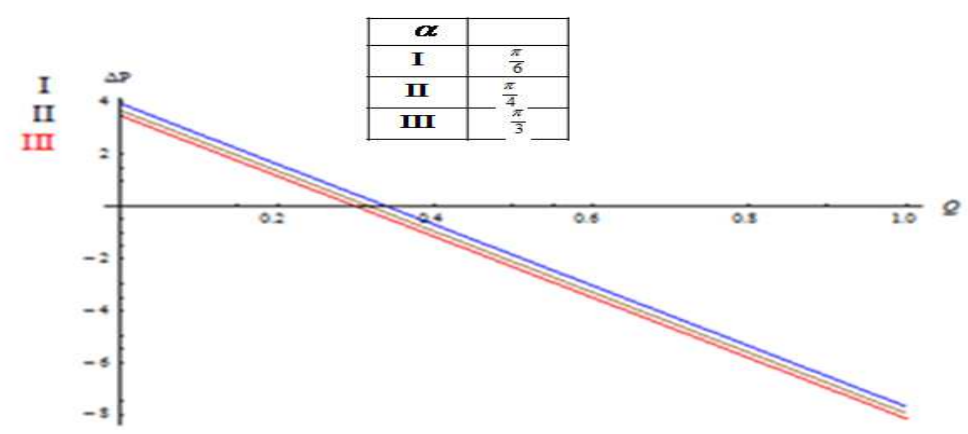

Figure 8: The Pressure Rise $\Delta P$ Variation with Mean Time of the Volumetric Flow Rate $\bar{Q}$ for Different Values of $\alpha$ With Fixed $\phi=0.6, \beta=0.3, \varepsilon=0.042, W e=3$ And $\eta=1.25$.

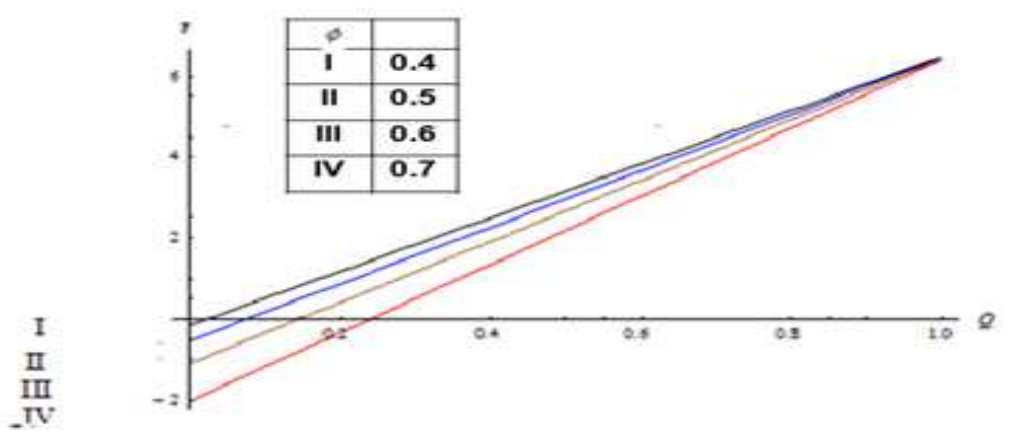

Figure 9: The Frictional Force $F$ Variation with Mean Time of the Volumetric Flow Rate $\bar{Q}$ for Different Values of $\phi$ With Fixed

$$
\beta=\mathbf{0 . 3}, \varepsilon=\mathbf{0 . 0 4}, W e=3 \alpha=\frac{\pi}{6} \text { and } \eta=1.25 \text {. }
$$

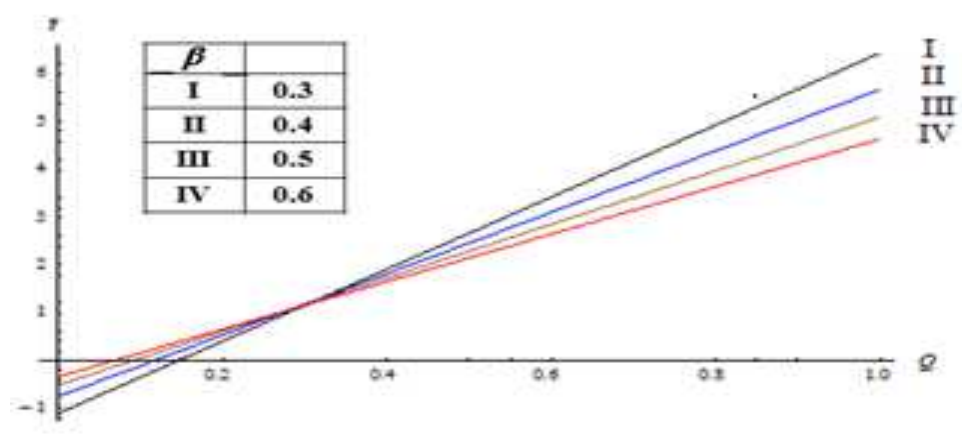

Figure 10: The Frictional Force $F$ Variation with Mean Time of the Volumetric Flow Rate $\bar{Q}$ for Different Values of $\beta$ With

Fixed $\phi=0.6, \varepsilon=0.04, W e=3 \alpha=\frac{\pi}{6}$ and $\eta=1.25$. 


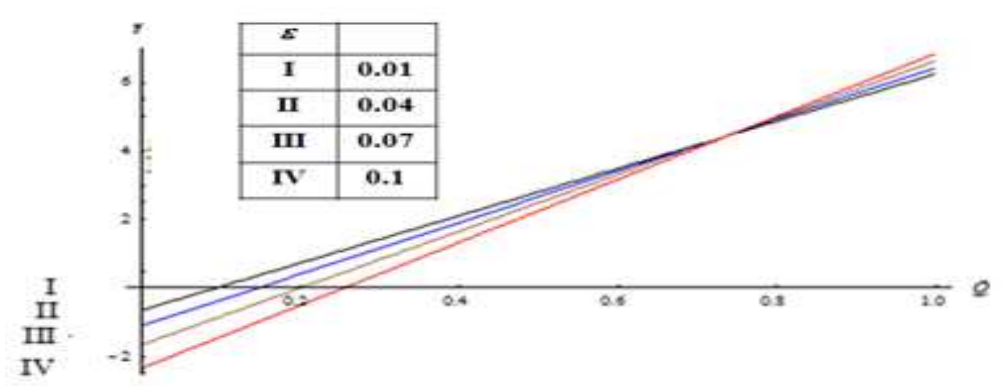

Figure 11: The Frictional Force $F$ Variation with Mean Time of the Volumetric Flow Rate $\bar{Q}$ for Different Values of $\varepsilon$ With Fixed $\phi=\mathbf{0 . 6}, \beta=\mathbf{0 . 3}, W e=\mathbf{3}, \alpha=\frac{\pi}{6}$ and $\eta=1.25$.

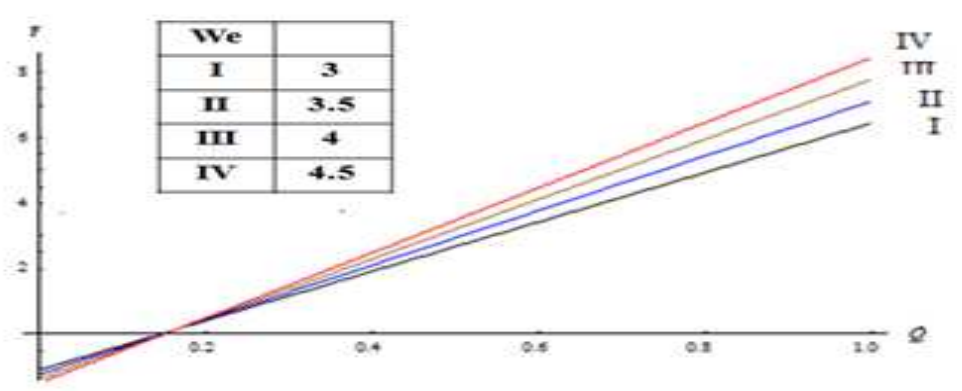

Figure 12: The Frictional Force $F$ Variation with Mean Time of the Volumetric Flow Rate $\bar{Q}$ for Different Values of We With Fixed $\phi=0.6, \beta=0.3 \varepsilon=0.04, \alpha=\frac{\pi}{6}$ and $\eta=1.25$.

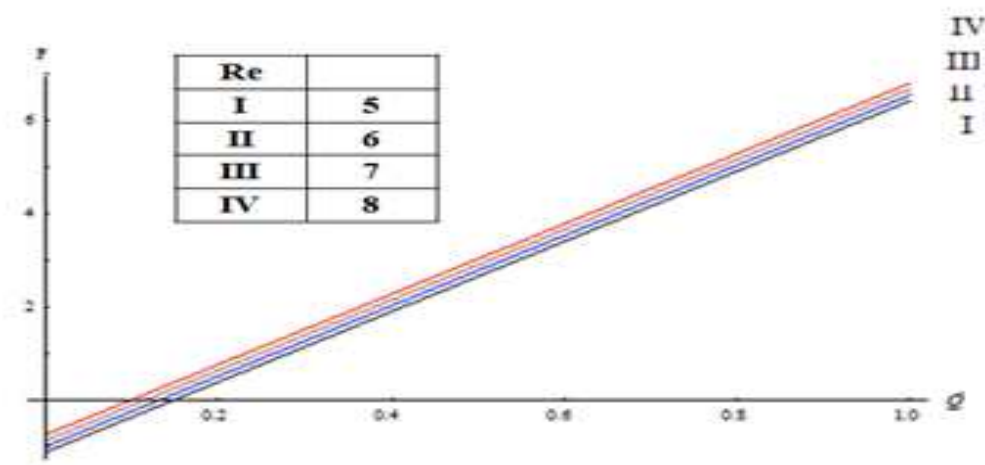

Figure 13: The Frictional Force $F$ Variation with Mean Time of the Volumetric Flow Rate $\bar{Q}$ for Different Values of Re With Fixed $\phi=0.6, \beta=0.3, \varepsilon=0.04, W e=3, \alpha=\frac{\pi}{6}$ and $F r=4$. 


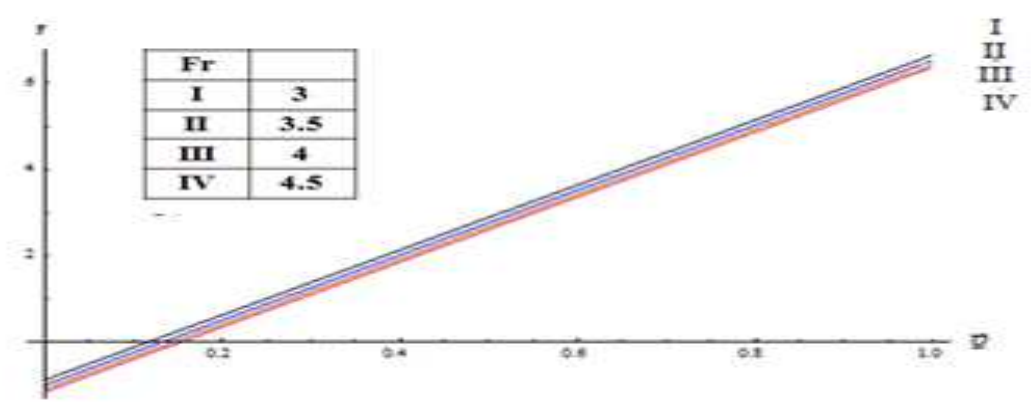

Figure 14: The Frictional Force $F$ Variation with Mean Time of the Volumetric Flow Rate $\bar{Q}$ for Different Values of $\operatorname{Fr}$ With Fixed $\phi=0.6$, $\beta=0.3, \varepsilon=0.04, W e=3, \alpha=\frac{\pi}{6}$ and $\operatorname{Re}=5$.

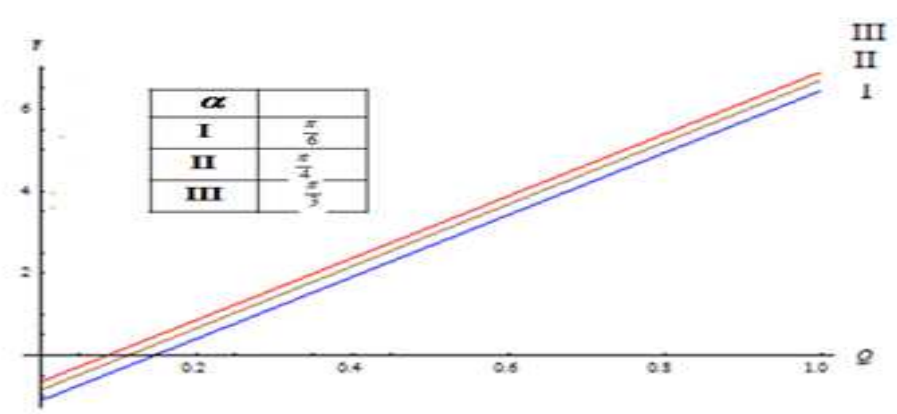

Figure 15: The Frictional Force $F$ Variation with Mean Time of the Volumetric Flow Rate $\bar{Q}$ for Different Values of $\alpha$ With

Fixed $\phi=0.6, \beta=0.3, \varepsilon=0.04, W e=3$ and $\eta=1.25$.

\section{CONCLUSIONS}

- The Variation in pressure rise $\Delta P$ with respective time average volumetric flow rate $\bar{Q}$ decreases as of permeability parameter ' $\beta$ ' increases.

- The Variation in pressure rise $\Delta P$ with respective time average volumetric flow rate $\bar{Q}$ increases as lining ' $\varepsilon$ ' increases.

- The Variation in pressure rise $\Delta P$ with respective time average volumetric flow rate $\bar{Q}$ decreases as inclination ' $\alpha$, increases.

- The Frictional force $F$ with respective time average volumetric flow rate $\bar{Q}$ increases some stage then decreases as of permeability parameter ' $\beta$ ' increases.

- The Frictional force $F$ with respective time average volumetric flow rate $\bar{Q}$ decreases some stage and then increases as lining ' $\mathcal{E}$ ' increases. 
- The Frictional force $F$ with respective time average volumetric flow rate $\bar{Q}$ increases as inclination ' $\alpha$, increases.

\section{ACKNOWLEDGEMENTS:}

We thank Prof. S. Sreenadh sir for his constant support, encouragement in preparing this Manuscript

\section{REFERENCES}

1. H.T. Tang and Y.C. Fung, Fluid movement with permeable walls covered by porous media. A model of lung alveolar sheet, J. Appl. Mech. 97 (1975) 4550.

2. S. N. Majhi and V. R. Nair, pulsatile flow of third grade fluids under body acceleration- modeling blood flow, Int. J. Engg. Sci... 32 (5) (1996) 839-846.

3. A. H. Shapiro, M. Y.Jaffrin, and S. L.Weinberg, peristaltic pumping with long wavelengths at low Reynolds number, J. Fluid Mech. 37 (1969) 799825.

4. V.P Srivastava, and L.M Srivastava, Effects of Poiseuille flow on peristaltic transport of a particulate suspension, J.Applied Math.Phys. 46 (1995) 655678.

5. K.Vajravelu, S. Sreenadh. And V.Ramesh Babu, peristaltic pumping of a Herschel Bulkley fluid in a channel. Applied Mathematics and Computation. 169 (2005) 726735.

6. M. Kothandapani and S. Srinivas, Non-linear peristaltic transport of a Newtonian fluid in an inclined asymmetrical channel through porous medium. Phys. Lett-A, 372 (2008) 12651276.

7. S.Srinivas and R.Gayatri, peristaltic transport of a Newtonian fluid in a vertical Computation, (215) (2009) 185196.

8. K. K Raju,. And R. Devanathan, peristaltic motion of a non-Newtonian Fluid. Rheol.Acta 11 (1972) 170-179.

9. J. C. Mishra and S. K.Ghosh, A mathematical model for the study of blood flow through a channel with permeable walls. Acta mechanica. 122 (1997) 137-153.

10. M. Misra and A. R. Rao, peristaltic transport of a Newtonian fluid in an asymmetric channel. Z Angew, Math. Phys. 54 (2003) $530-550$

11. Abd. El Hakeem, Abd. El Naby and A.E.M El Misery. Effects of endoscope and generalized Newtonian fluid on peristaltic motion. Applied Mathematics and computation. 128 (1) (2002) 19-35.

12. A. Ebaid. Effects of magnetic field and wall slip condition on the peristaltic transport of a Newtonian fluid in an asymmetric channel. Phys. letters A. 372 (24) (2008) 4493-4499.

13. K. S. Mekheimer, peristaltic transport of a couple stress fluid in uniform and non uniform channels. Biorheology 39 (6) (2002) $755-765$.

14. A. M. Sobh, Interactions of couple stresses and slip flow on peristaltic transport in uniform and non-uniform channels. Turkish Journal of Engineering Environmental Sciences. 32 (2008) 117123.

15. K.Vajravelu, S.Sreenadh, K.Rajanikanth, Changhoon Lee, Peristaltic transport of a Williamson fluid in asymmetric channels with permeable walls, Nonlinear Analysis: Real World Applications 13 (2012), 28042822.

16. B.Jayaramireddy, M.V.Subba Reddy, C.Nadhamuni Reddy and P.Yogeswar reddy, Peristaltic flow of a Williamson fluid in an inclined planar channel under the effect of a magnetic field, Advances in Applied Science Research, 3 (1) (2012) 452461. 
17. E. F. ElShehawey and S. Z. A. Husseney, Effects of porous boundaries on peristaltic transport through a porous medium, Acta Mechanica, 143 (2000) 165177.

18. E. F. Elshehawey, N.T. Eldabe, E.M. Elghazy, A.Ebaid, peristaltic transport in an asymmetric channel through a porous medium. Applied Mathematics Computation. 182 (1) (2006) 140-150.

19. K. Ramesh and M. Devakar. Peristaltic transport of MHD Williamson fluid in an inclined asymmetric channel through porous medium with heat transfer. J.Cent. South univ. 22 (2015) 3189-3201.

20. R.Ellahi, M. M.Bhatti, and Arshad. Kiaz, Effects of magneto- hydrodynamics on peristaltic flow of Jeffery fluid in a rectangular duct through a porous media, Journal of porous media. 17 (2014) 143-157.

21. Manoranjan Mishra and A. Ramachandra Rao, Peristaltic transport with a Porous peripheral layer: model of a flow in gastro intestinal tract,Int. J. of. Biomechanics, 38 (4) (2005) 779789.

22. S.Sreenadh, S.Rajender, S.V.H.N. Krishna Kumari and Y.V.K. Ravi Kumar. Flow of Herschel-Bulkley fluid in an inclined flexible channel lined with porous material under peristalsis. Int. J. of Innovative Technology and Creative Engineering, 1 (7) (2011).

23. G.C.Sankad and Asha Patil. Impact of permeable Lining of the wall on the Peristaltic Flow of Herschel Bulkley Fluid in Applications and Applied Mathematics an International Journal, 11 (2) (2016) 663679.

24. B.Reddappa, A.Parandhama, S.Sreenadh, Peristaltic transport of conducting Williamson fluid in a Porous channel, J.Math.Comput.sci. 10 (2) (2020) 277288.

25. M.M. Bhatti, A.Riaz, L.Zhang, Sadiq M Sait \& R. Ellahi. Biologically inspired thermal transport on the rheology of Williamson hydromagnetic nanofluid flow with convection: an entropy analysis Journal of Thermal Analysis and Calorimetry (2020). 